\title{
Loss of keratin 13 in oral carcinoma in situ: a comparative study of protein and gene expression levels using paraffin sections
}

\author{
Hiroko Ida-Yonemochi ${ }^{1,2,3}$, Satoshi Maruyama ${ }^{3}$, Takanori Kobayashi ${ }^{3}$, \\ Manabu Yamazaki ${ }^{1}$, Jun Cheng $^{1}$ and Takashi Saku ${ }^{1,3}$ \\ ${ }^{1}$ Division of Oral Pathology, Department of Tissue Regeneration and Reconstruction, Niigata University \\ Graduate School of Medical and Dental Sciences, Niigata, Japan; ${ }^{2}$ Division of Anatomy and Cell Biology \\ of the Hard Tissue, Niigata University Graduate School of Medical and Dental Sciences, Niigata, \\ Japan and ${ }^{3}$ Oral Pathology Section, Department of Surgical Pathology, Niigata University Hospital, \\ Niigata, Japan
}

\begin{abstract}
Immunohistochemical loss of keratin (K)13 is one of the most valuable diagnostic criteria for discriminating carcinoma in situ (CIS) from non-malignancies in the oral mucosa while K13 is stably immunolocalized in the prickle cells of normal oral epithelium. To elucidate the molecular mechanism for the loss of K13, we compared the immunohistochemical profiles for K13 and K16 which is not expressed in normal epithelia, but instead enhanced in CIS, with their mRNA levels by in-situ hybridization in formalin-fixed paraffin sections prepared from $23 \mathrm{CIS}$ cases of the tongue, which were surgically removed. Reverse transcriptase-PCR was also performed using RNA samples extracted from laser-microdissected epithelial fragments of the serial paraffin sections in seven of the cases. Although more enhanced expression levels for K16 were confirmed at both the protein and gene levels in CIS in these seven cases, the loss of K13 was associated with repressed mRNA levels in four cases, but not in the other three cases. The results suggest that the loss of K13 is partly due to its gene repression, but may also be due to some unknown post-translational events.
\end{abstract}

Modern Pathology (2012) 25, 784-794; doi:10.1038/modpathol.2011.218; published online 3 February 2012

Keywords: in-situ hybridization; keratin 13; laser-microdissection; oral carcinoma in situ; RT-PCR

\section{Introduction}

One of the primary histopathological findings of oral squamous epithelial lesions is disturbance of keratinization toward the epithelial surface, known as dyskeratosis, which can be identified on hematoxylin and eosin (HE)-stained sections. ${ }^{1}$ On the other hand, there are some other keratinocytic differentiation disorders which are not easily recognized with routine HE staining, but which are clearly revealed by immunohistochemistry for keratin (K) subtypes. For instance, in normal epithelia of the oral mucosa, the first basal cells express K19, whereas the prickle

Correspondence: Professor T Saku, Division of Oral Pathology, Department of Tissue Regeneration and Reconstruction, Niigata University Graduate School of Medical and Dental Sciences, 25274 Gakkocho-dori, Chuoku, Niigata 951-8514, Japan.

E-mail: tsaku@dent.niigata-u.ac.jp

Received 19 July 2011; revised 19 December 2011; accepted 21 December 2011; published online 3 February 2012 cells in the third basal and upper layers express $\mathrm{K} 13 .^{2-4}$ As parabasal (second basal) cells neither express K13 nor K19, but are positive for Ki-67, the parabasal cells may comprise a cell proliferating center. Expansions of the Ki-67-positive $(+)$ cell proliferating center start to replace the first basal cells in the lower direction, as well as the prickle cells in the upper direction, resulting in gradual losses of K19 and K13 in those cells, which should represent such neoplastic conditions as epithelial dysplasia and carcinoma in situ (CIS). ${ }^{5}$ In particular, a total loss of K13 in the prickle cell layer should be one of the most important histochemical markers for the diagnosis of differentiated types of oral CIS, because the prickle cells of CIS are unable to be distinguished from the normal prickle cells on HE sections. ${ }^{4-6}$

$\mathrm{K} 13$, an acidic keratin, is assembled with $\mathrm{K} 4$, its basic partner, to form intermediate cytoskeletal filaments of most internal stratified epithelia. ${ }^{7}$ 
As K13 has been mainly immunolocalized from the third basal layer up to the surface in normal oral mucosa, the loss of $\mathrm{K} 13$ is considered to be related with the severity of dysplastic changes. ${ }^{4-6,8-14}$ Regarding the disappearance of K13 in laryngeal squamous cell carcinomas (SCCs) with definite keratinization tendencies, masking or modification of K13 antigenic sites due to some dysfunction of the K13 gene has been suggested. ${ }^{11}$ However, there has been no investigation on the K13 gene expression levels in the oral squamous epithelial lesions lacking the immunohistochemical expression of the K13 protein.

To understand molecular mechanisms for the loss of K13 protein in oral neoplastic conditions, we wanted to determine its gene transcription status by in-situ hybridization (ISH), and as well as by reverse transcriptase (RT)-PCR using K16 as a control. In contrast to K13, K16 is not expressed (-) in normal oral epithelia, whereas its expression levels are enhanced in neoplastic conditions. ${ }^{15} \mathrm{~K} 16$ has been reported to be a marker for activated keratinocytes or their hyperproliferative conditions not only in the oral cavity, but in other organs. ${ }^{16,17}$

For RT-PCR analyses of surgical specimens, it is preferable to use fresh cells or tissue samples. However, it is difficult to dissect fresh epithelial tissue samples from oral squamous epithelial lesions, because it is unknown which parts are immunohistochemically positive for K13 at the time of surgery. Therefore, in this study, we utilized the laser-capture microdissection (LMD) technique to obtain relatively pure epithelial cell samples from paraffin sections of surgical materials, which were immunohistochemically confirmed by referring to the presence or absence of $\mathrm{K} 13$ in sections consecutive to those selected for LMD. As RNA extraction from LMD-prepared, formalin-fixed paraffin section fragments had not been established, we developed an efficient extraction method for this particular purpose.

\section{Materials and methods}

\section{Materials}

Twenty-three surgical materials of oral CIS of the tongue were selected from the surgical pathology files in the Division of Oral Pathology, Niigata University Graduate School of Medical and Dental Sciences, during the 4-year period from 2003 to 2006. All of the specimens were routinely fixed in $10 \%$ formalin and embedded in paraffin. Serial $5-\mu \mathrm{m}$ sections were cut from the paraffin blocks. The serial sections were stained with HE and immunohistochemically for K13 and K16. They were also used for ISH for K13, as well as for LMD. The experimental protocol for analyzing surgical materials was reviewed and approved by the Ethical Board of Niigata University Graduate School of Medical and Dental Sciences (Oral Life Science).

\section{Antibodies}

Monoclonal mouse antibody to human K13 (clone DE-K13, IgG ${ }_{1}$, diluted at 1:200) was purchased from Dako (Glostrup, Denmark). A mouse monoclonal antibody against K16 (clone LL025, IgM, diluted at 1:40) was obtained from Abcam (Cambridge, UK).

\section{Immunohistochemistry}

The ChemMate Envision System (Dako) was used for immunohistochemical staining. Prior to incubation with the primary antibodies, sections were autoclaved in $0.01 \mathrm{M}$ citrate buffer ( $\mathrm{pH}$ 6.0) for $10 \mathrm{~min}$ at $121^{\circ} \mathrm{C}$ and then kept standing for $20 \mathrm{~min}$ at room temperature. To block endogenous peroxidase activities, all sections were quenched with $0.3 \%$ hydrogen peroxide in $100 \%$ methanol for $30 \mathrm{~min}$ at room temperature and rinsed with PBS containing $0.5 \%$ milk protein (Morinaga Milk Industry, Tokyo, Japan) and $0.05 \%$ Triton X-100 (T-PBS), and then they were incubated with $5 \%$ milk protein in T-PBS for $1 \mathrm{~h}$ at room temperature to block non-specific protein-binding sites. After incubation with the primary antibodies overnight at $4{ }^{\circ} \mathrm{C}$, the sections were rinsed in T-PBS and then treated with polymerimmune complexes (EnVision + peroxidase, rabbit/ mouse; Dako, 1:1) for $1 \mathrm{~h}$ at room temperature. After rinsing with T-PBS, peroxidase reaction products were visualized by incubation with $0.02 \% 3^{\prime} 3$ diaminobenzidine (Dojindo Laboratories, Kumamoto, Japan) in $0.05 \mathrm{M}$ Tris-HCl (pH 7.6) containing $0.005 \%$ hydrogen peroxide. For control experiments, the primary antibodies were replaced with pre-immune mouse $\operatorname{IgG}_{1} \cdot{ }^{5}$

\section{Preparation of RNA Probes}

The human K13 cDNA was amplified by RT-PCR, using RNA samples of normal human oral mucosa, and $5^{\prime}$-TTGAA AACAA CCGGG TCATC-3' (sense) and $5^{\prime}$-GCTCT TCATT CAGGC TCTCG- $3^{\prime}$ (antisense) primers. The PCR products $(195 \mathrm{bp})$ were subcloned into plasmid vectors (pBluescript II, Promega, Madison, WI, USA) and were digested with $\mathrm{NcoI}$ and SalI. The linearized plasmids were used as templates to synthesize digoxigenin-labeled RNA antisense probes by T7 RNA polymerase (Promega) and sense probes by SP6 RNA polymerase (Promega).

\section{In-Situ Hybridization}

After deparaffinization, the sections were washed in $2 \times$ standard saline citrate (SSC) and treated with $5 \mu \mathrm{g} / \mathrm{ml}$ of proteinase K (Sigma Chemical, St Louis, MO, USA) for $20 \mathrm{~min}$ at $37^{\circ} \mathrm{C}$. They were then washed with $0.2 \%$ glycine in PBS, fixed with $4 \%$ paraformaldehyde in $0.1 \mathrm{M}$ phosphate buffer $(\mathrm{pH}$ 7.5) for $5 \mathrm{~min}$, dehydrated with a series of ethanol $(70-100 \%)$ and air-dried. Hybridization was 
performed for $15 \mathrm{~h}$ at $43{ }^{\circ} \mathrm{C}$ in a moist chamber. The hybridization solution contained $10 \%$ dextran sulfate, $1 \times$ Denhardt's solution, $100 \mu \mathrm{g} / \mathrm{ml}$ of salmon sperm DNA, $125 \mu \mathrm{g} / \mathrm{ml}$ of yeast tRNA, $3 \times$ SSC, $50 \%$ formamide, and $1 \mu \mathrm{g} / \mathrm{ml}$ of probes in $10 \mathrm{mM}$ phosphate buffer ( $\mathrm{pH}$ 7.4). After hybridization, the sections were rinsed with $0.1 \times$ SSC and $0.2 \mathrm{M}$ maleic acid buffer ( $\mathrm{pH}$ 7.5), and incubated for $1 \mathrm{~h}$ with alkaline phosphatase-conjugated sheep antidigoxigenin antibody (Roche Diagnostics $\mathrm{GmbH}$, Mannheim, Germany). Alkaline phosphatase products were developed with NBT/BCIP stock solution (diluted at 1:50, Roche), and the sections were counterstained with methyl green as described previously. ${ }^{18}$

\section{Total RNA Extraction from Paraffin Sections}

For RNA extraction from whole paraffin sections, five serial $5-\mu \mathrm{m}$-thick sections were prepared from each paraffin-embedded tissue block and placed in $1.5 \mathrm{ml}$ sterile microtubes. To avoid cross-contamination of samples, a new microtome blade was used for every block. The area of the microtome around the blade was cleaned with $70 \%$ ethanol every time paraffin blocks were replaced. The sections were incubated at $98{ }^{\circ} \mathrm{C}$ for $10 \mathrm{~min}$ in $200 \mu \mathrm{l}$ of lysis buffer (20 mM Tris-HCl, pH 8.0, 20 mM EDTA, 2\% SDS) without deparaffinization with xylene. To the tubes, $100 \mu \mathrm{g}$ of proteinase $\mathrm{K}$ was added and incubated at $60^{\circ} \mathrm{C}$ for $1 \mathrm{~h}$. A 1 -ml volume of TRIzol reagent (Gibco, Invitrogen, Carlsbad, CA, USA) was added to the samples, and total RNA was extracted according to the manufacturer's instruction. The RNA pellet was reconstituted in $10 \mu \mathrm{l}$ of $0.05 \%$ diethyl pyrocarbonate-treated water.

\section{LMD and Total RNA Extraction}

Seven specimens that contained both of $\mathrm{K} 13(+) /$ $\mathrm{K} 16(-)$ normal epithelial areas and $\mathrm{K} 13(-) / \mathrm{K} 16(+)$ CIS areas in the same sections were selected for LMD. A total of 10 serial paraffin sections were cut at a thickness of $5 \mu \mathrm{m}$ and mounted on slide glasses to which thin film sheets (Meiwa Shoji, Tokyo, Japan) had been attached by silicone adhesive (GE Toshiba Silicon, Tokyo, Japan). The sections were deparaffinized by two changes of xylene for $10 \mathrm{~min}$, rehydrated in 100,90 , and $70 \%$ ethanol for $30 \mathrm{~s}$ each, and stained with 1\% toluidine blue solution. A Leica AS LMD microdissection system with a UV laser (Leica Microsystems GmbH, Wetzlar, Germany) was used to recover epithelial parts from the tissue sections prepared as mentioned above. We separately captured $\mathrm{K} 13(+)$ normal epithelial parts, as well as K13(-) CIS parts, which were immunohistochemically and respectively confirmed in their consecutive sections. Total RNA was extracted from laser-captured samples by using the TRIzol reagent as described above.

\section{Reverse Transcriptase-PCR}

After DNase I (1 U; Invitrogen) treatment of total RNA samples, cDNAs were synthesized with the SuperScript First-Strand Synthesis System (Invitrogen). Following the RT, PCR was carried out in an Astec thermal cycler PC-800 (Astec, Fukuoka, Japan). Oligonucleotide primers flanking the human K13 gene (nucleotide number 539-733, Number BC126184.1, GeneBank) were synthesized using primer sets as follows: $5^{\prime}$-TTGAA AACAA CCGGG TCATC- ${ }^{\prime}$, forward, and $5^{\prime}$-GCTCT TCATT CAGGC TCTCG $-3^{\prime}$, reverse, to generate a 195-bp product. K16 primers were as follows: $5^{\prime}$-TCTTG CTGGA TGTGA AGACG- $3^{\prime}$, forward, and 5'-GAAGC TGGAT GAGCT CTGCT 3', 197-bp product (nucleotide number 12191415, Number AF061812, GeneBank). For the competitive PCR, we also synthesized oligonucleotide primers of human $\beta$-actin as follows: $5^{\prime}$-TCACC CACAC TGTGC CCATC TACGA- $3^{\prime}$, forward, and $5^{\prime}$ CAGCG GAACC GCTCA TTGCC AATGG-3', reverse, to generate a 295-bp product (nucleotide number 549-843, Number NM001101, GeneBank). The thermocycling protocol during the 40-45 amplification cycles was as follows: denaturation at $94{ }^{\circ} \mathrm{C}$ for $45 \mathrm{~s}$, annealing at $58{ }^{\circ} \mathrm{C}$ for $45 \mathrm{~s}$, extension at $72{ }^{\circ} \mathrm{C}$ for $45 \mathrm{~s}$, and termination with a final cycle of extension at $72{ }^{\circ} \mathrm{C}$ for $7 \mathrm{~min}$. The amplified DNA fragments were analyzed by electrophoresis on $3 \%$ agarose gels.

\section{Direct Sequencing of PCR Products}

The 195-bp PCR products for K13 from LMD samples were sequenced to confirm the presence of K13 mRNA, using the forward primer described above. All PCR products were extracted from the agarose gels and subjected to cycle sequencing by using the ABI PRISM BigDye Terminator version 3.1 Cycle Sequencing Kit (Applied Biosystems, Foster City, CA, USA). The reaction products were then applied in an ABI PRISM 310 Genetic Analyzer (Applied Biosystems) and the sequencing data were analyzed by using the DNA sequencing analysis software, version 3.0 (Applied Biosystems).

\section{Results}

\section{Immunohistochemical Profiles for K13 and K16 in Oral Epithelial Lesions}

Immunohistochemical profiles for K13 and K16 were compared among four categories of oral epithelial conditions; normal, dysplasia, CIS, and SCC. In normal epithelia (Figure 1a), K13 was distinctly localized in the epithelial layer, except for the basal cells (Figure 1b), whereas there were no positivities for K16 in any part of the epithelial layer (Figure 1c). Normal epithelia were also confirmed by the presence of K19 in the first basal cells and Ki-67-positive $(+)$ cells in the second basal layer as described previously (not shown). ${ }^{5}$ 

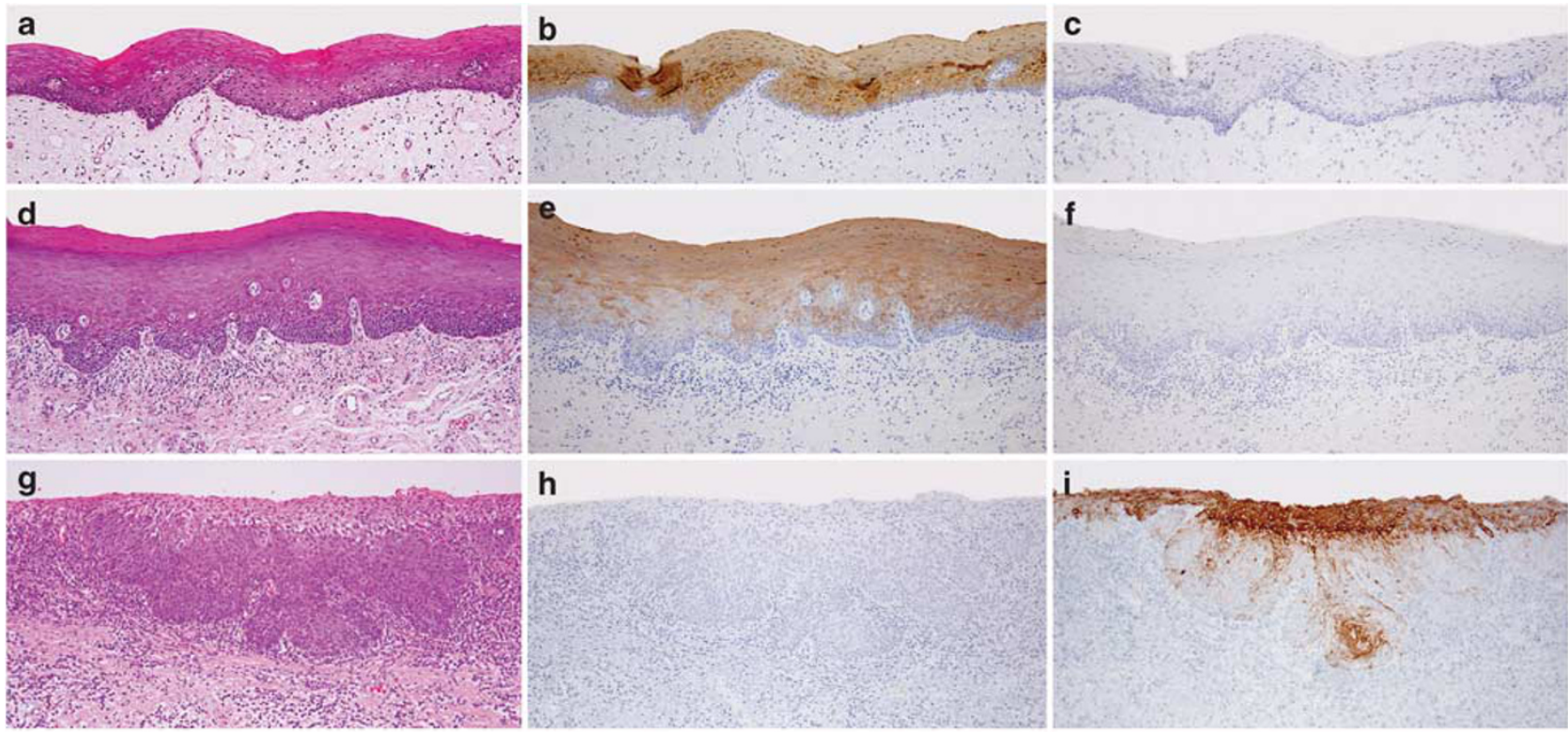

h
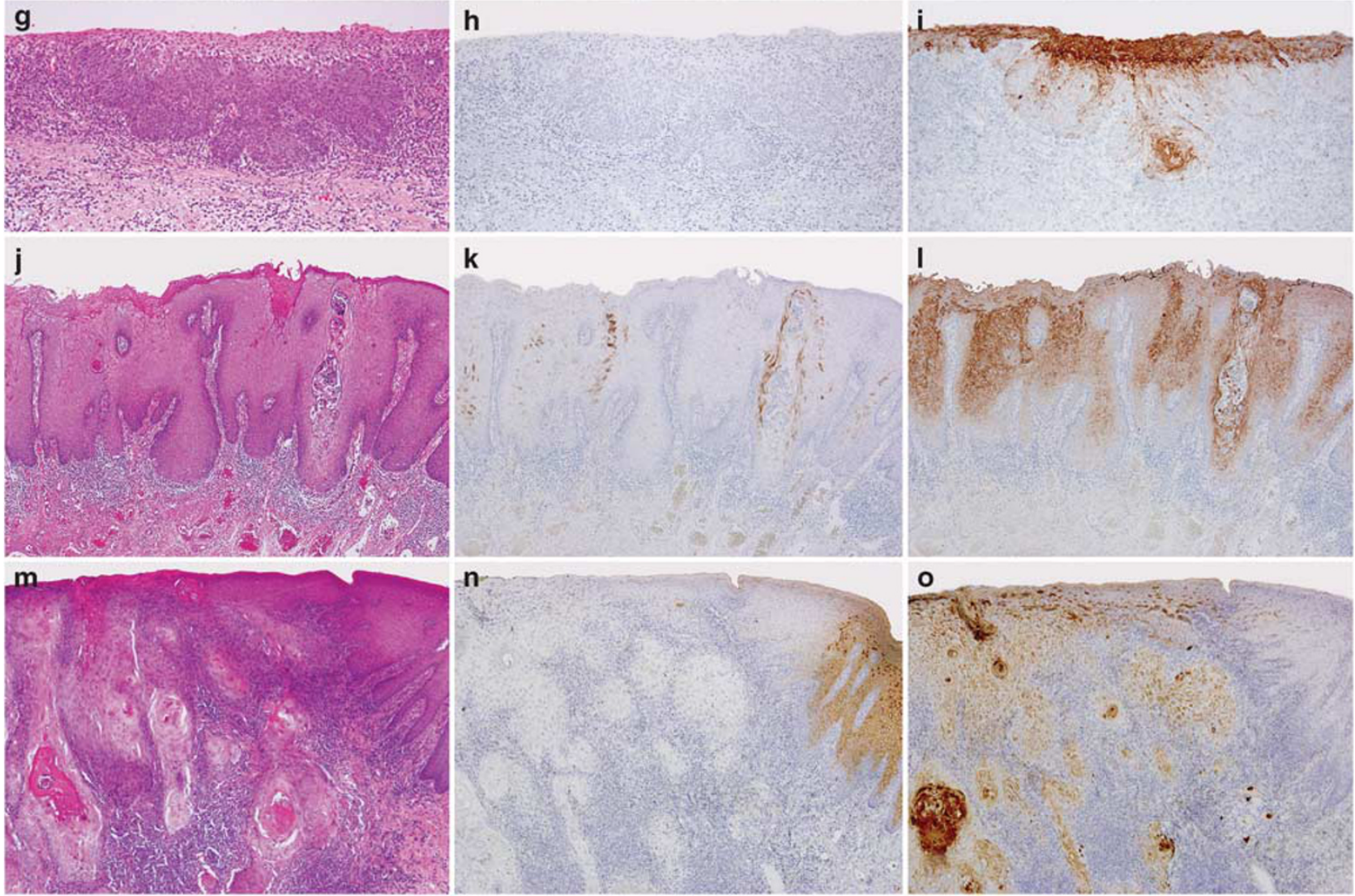

Figure 1 Histopathological and immunohistochemical profiles of normal epithelia and epithelial lesions of the oral mucosa. (a-c) Normal epithelium; (d-f) epithelial dysplasia; (g-i) carcinoma in-situ (CIS), basaloid type; (j-l) CIS, differentiated type; (m-o) squamous cell carcinoma (SCC). (a, d, g, j, m) Hematoxylin and eosin (HE) stain; (b, e, h, k, n) immunoperoxidase stain for keratin (K)13; immunoperoxidase stain for K16 (c, f, i, l, o), hematoxylin counterstain. (a-f, j-o) $\times 100$; $(\mathbf{g}-\mathbf{i}) \times 200$ magnification. Normal and dysplastic epithelia were immunohistochemically defined by the absence of K16 and the presence of K13 in the prickle cells (a-f). In contrast, two types of CIS, basaloid ( $(\mathrm{g}-\mathbf{i})$ and differentiated $(\mathbf{j}-\mathbf{l})$, were not yet invasive, whereas its immunohistochemical features were the same as those of SCC: no K13 (k, n) positive, but K16 positive (l, o).

Mild and moderate epithelial dysplasia (Figure 1d) showed basically similar staining patterns to those of normal epithelia for K13 (Figure 1e) and K16 (Figure 1f). In dysplasia, K19 disappears from the basal cells, and Ki-67 + cells were expansively located towards the first and third basal and upper layers (not shown). ${ }^{5}$ Two types of CIS were demonstrated in Figure 1: basaloid type (Figure 1g-i) and differentiated type (Figure 1j-1). ${ }^{5}$ CIS is not yet invasive, whereas its immunohistochemical features are the same as those of SCC (Figure 1m-o). Neither
K13 (Figure 1k and n) nor K19 (not shown) were positive, whereas K16 (Figure 1l and o) and K17 (not shown) were reciprocally positive with an expansion of Ki-67 + cells including the first basal layer (not shown). ${ }^{6}$

\section{RNA Extraction from Formalin-fixed, Paraffin-Embedded Sections}

The efficiency of RNA extraction from formalinfixed paraffin sections is summarized in Table 1. 
Table 1 The efficiency of RNA extraction from formalin-fixed paraffin sections

\begin{tabular}{lcr}
\hline & Whole sections & $L M D$ \\
\hline Amount of sections $(\mu \mathrm{m}$ thick $\times$ slices $)$ & $5 \times 5$ & $5 \times 10$ \\
Total size of samples $\left(\mathrm{mm}^{2}\right)$ & $170.1(16-375)$ & $4.1(1-7.5)$ \\
RNA concentration $\left(\mu \mathrm{g} / \mathrm{ml}^{2}\right)$ & $495.6(59.3-3418.1)$ & $209.0(13.6-594.3)$ \\
Total RNA amount $(\mu \mathrm{g})$ & $4.5(0.59-30.8)$ & $1.9(0.1-5.3)$ \\
OD $_{260 / 280}$ ratio & $1.1(0.71-1.48)$ & $0.9(0.6-1.3)$ \\
\hline
\end{tabular}

Abbreviations: LMD, laser-capture microdissection; OD, optical density.

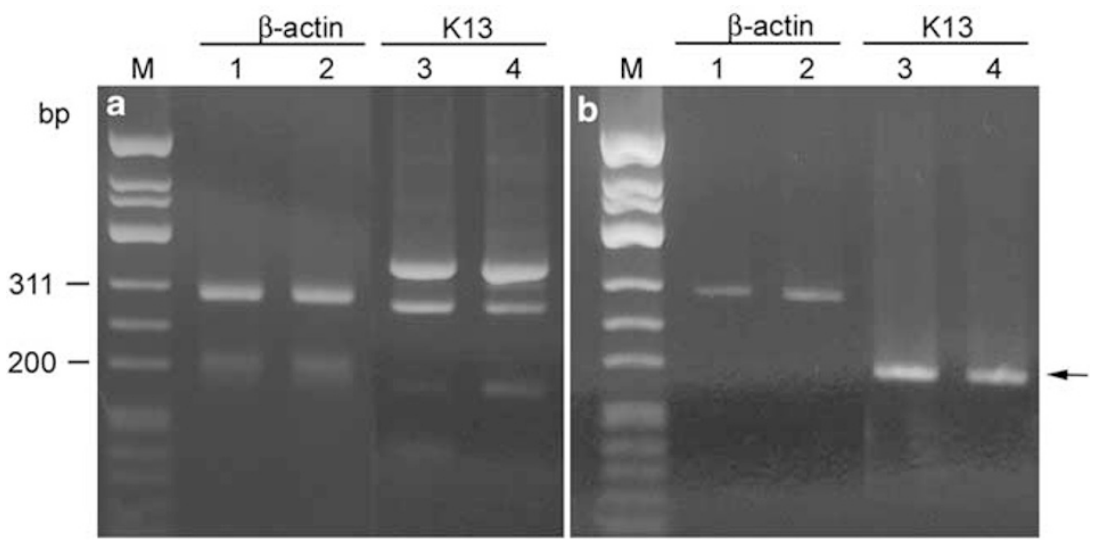

Figure 2 Reverse transcriptase (RT)-PCR for keratin (K)13 mRNA with or without DNase I treatment. (a) Without DNase I treatment, (b) with DNase I treatment. (Lanes 1 and 2) 295-bp fragment of $\beta$-actin, (lanes 3 and 4) 195-bp fragment of K13. (Lanes 1 and 3) Case 1, (lanes 2 and 4) case 2. PCR product of $195 \mathrm{bp}$ showed K13 mRNA (arrow), and other extra bands implied DNA contamination (lanes 3 and 4 ). Without DNase I treatment, the band of K13 mRNA was very faint (a); however, after DNA degradation, the target mRNA band became clear (b).

The total RNA amount from five whole sections, which had an average tissue area of $170.1 \mathrm{~mm}^{2}$, was $4.5 \mu \mathrm{g}$. On the other hand, that from 10 epithelial part fragments prepared by LMD was $1.9 \mu \mathrm{g}$. As DNA contamination was suspected because of low ratio of $\mathrm{OD}_{260 / 280}$ and very faint bands of K13 mRNA in the step of RNA purification (Figure 2a), before cDNA syntheses, the RNA samples were treated with DNase I after total RNA extraction by usual technique using TRIzol reagent. By DNA degradation, the target K13 mRNA band became clearer (Figure 2b). As amplicons generated by the primers for K13 mRNA contained the intron sequences, it was possible to distinguish whether PCR products were DNA or RNA by judging their sizes. PCR products of $195 \mathrm{bp}$ indicated K13 mRNA, whereas 394-bp products implied DNA contamination, as did the presence of extra bands (Figure 2a). From these results, we concluded that DNase I treatment was essential in RT-PCR for samples extracted from formalin-fixed paraffin sections.

\section{RT-PCR for K13 and K16 of Samples from Whole Paraffin Sections}

We selected two specimens from the same surgical materials that contained CIS regions. One specimen contained only normal epithelium (Figure 3a), which showed K13 positivity (Figure 3c) and no K16 positivity (Figure 3e). The other specimen had a CIS region (Figure 3b), which did not show K13 immunoreaction (Figure 3d), but was partially immunopositive for K16 (Figure 3f). We made each set of five serial paraffin sections from these two specimens and extracted RNA from the whole sections containing these lesions. Before the RTPCR experiment, we confirmed immunohistochemically that the subepithelial areas of these sections did not contain $\mathrm{K} 13(+)$ or $\mathrm{K} 16(+)$ epithelial components to avoid contamination by nonsurface epithelial cells. To ascertain that each RNA sample could yield RT-PCR products, amplification was performed using ubiquitously expressed $\beta$-actin primers that should amplify a 295-bp $\beta$-actin mRNA (Figure 4; lanes 1 and 2). From the $\mathrm{K} 13(+)$ epithelium (Figure 3c), we detected a clear band of 195-bp K13 mRNA (Figure 4; lane 3); however, the band was also seen in the non-K13-immunopositive CIS regions (areas indicated by arrows in Figure 3d, Figure 4; lane 4). On the other hand, obvious mRNA expression of K16 was only detected from the K16immunopositive area (Figure $3 \mathrm{e}$ and f, Figure 4; lanes 5 and 6). 

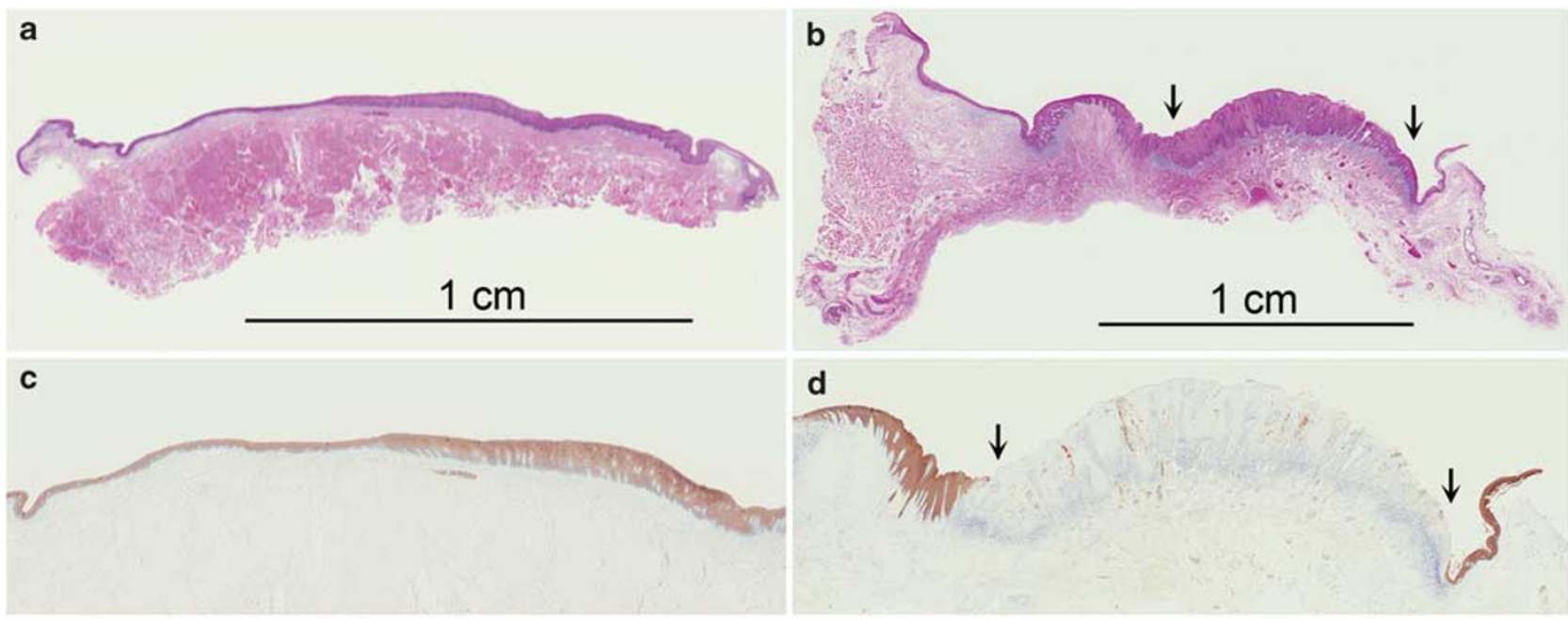

e

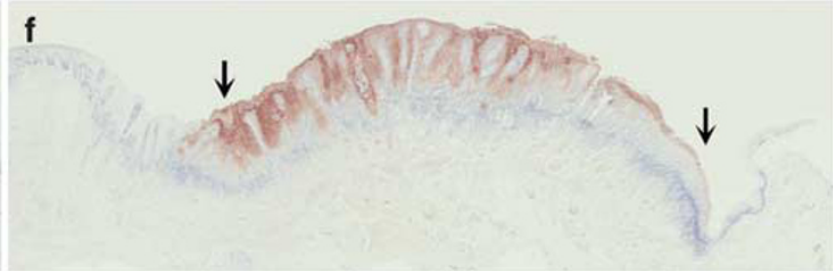

Figure 3 Comparative immunohistochemical profiles of normal epithelium vs carcinoma in situ (CIS) within the same surgical specimen. (a, b) Hematoxylin and eosin (HE) stain, (c, d) immunoperoxidase stain for keratin (K)13, (e, f) immunoperoxidase stain for K16, hematoxylin counterstain. (a, c, e) Normal oral epithelium, (b, d, f) CIS lesion (indicated by arrows). Normal epithelium was shown to be K13 immunopositive (c) and have no K16 immunopositive (e) stainings. On the other hand, CIS region did not show K13 immunoreaction (d), but was partially immunopositive for K16 (f). K13 and K16 immunopositive epithelial were not present in the submucosal layer.

\section{RT-PCR for K13 from Microdissected Epithelial Fragments}

From seven surgical specimens, in which both normal and CIS epithelial parts were contained within the same sections (Figure 5a and b), serial sections were immunohistochemically examined for K13. Normal epithelial parts were definitely K13 positive $(\mathrm{K} 13(+)$; Figure 5c and e), whereas CIS parts showed no K13 positivity (K13(-); Figure 5d and f). Using LMD, we recovered $\mathrm{K} 13(+)$ and $\mathrm{K} 13(-)$ parts separately by referring to the immunohistochemical results in consecutive sections (Figure $5 \mathrm{~g}$ and $\mathrm{h}$ ). RNA samples were extracted from the captured epithelial cell sheets and were further processed for standard RT-PCR reactions. Strong bands for the gene of $\beta$-actin, as a housekeeping gene, were obtained after 40 cycles of amplification (Figure 6). We LMD captured K13(-) CIS parts from seven specimens and compared their K13 mRNA expression levels with K13(+) normal epithelial parts. In four cases $(57 \%)$, there were strong bands of K13 mRNA even in the K13(-) CIS regions (Figure 6a), whereas in the other three cases $(43 \%)$, only faint bands were obtained, suggesting their lowered levels of K13 mRNA expression (Figure 6b).

In the present study, we used conventional competitive RT-PCR method instead of real-time PCR to compare the gene expression levels, because

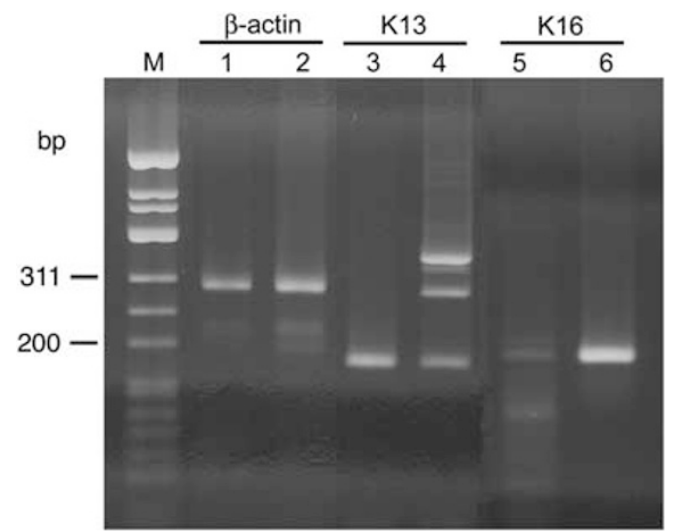

Figure 4 Reverse transcriptase (RT)-PCR for keratin (K)13 and K16 mRNA taken from whole paraffin sections. (Lanes 1 and 2) 295-bp fragment of $\beta$-actin, (lanes 3 and 4) 195-bp fragment of K13, (lanes 5 and 6) 197-bp fragment of K16. (Lanes 1, 3 and 5) K13 positive $(+)$ and no K16 positive (-) normal epithelial samples, (lanes 2, 4 and 6) K13(-) and K16(+) CIS samples. To ascertain that each RNA sample could yield RT-PCR products, amplification was performed using ubiquitously expressed $\beta$-actin primers that should amplify a 295-bp $\beta$-actin mRNA (lanes 1 and 2). From the K13( + ) epithelium, we detected a clear band of 195-bp K13 mRNA (lane 3); however, the band was also seen in the K13(-) carcinoma in situ (CIS) regions (lane 4). On the other hand, enhanced mRNA expression of K16 was only detected from the $\mathrm{K} 16(+)$ area (lane 6).

we had to distinguish the PCR products from the contamination of genomic DNA by different-sized bands in the same lanes as described above. 

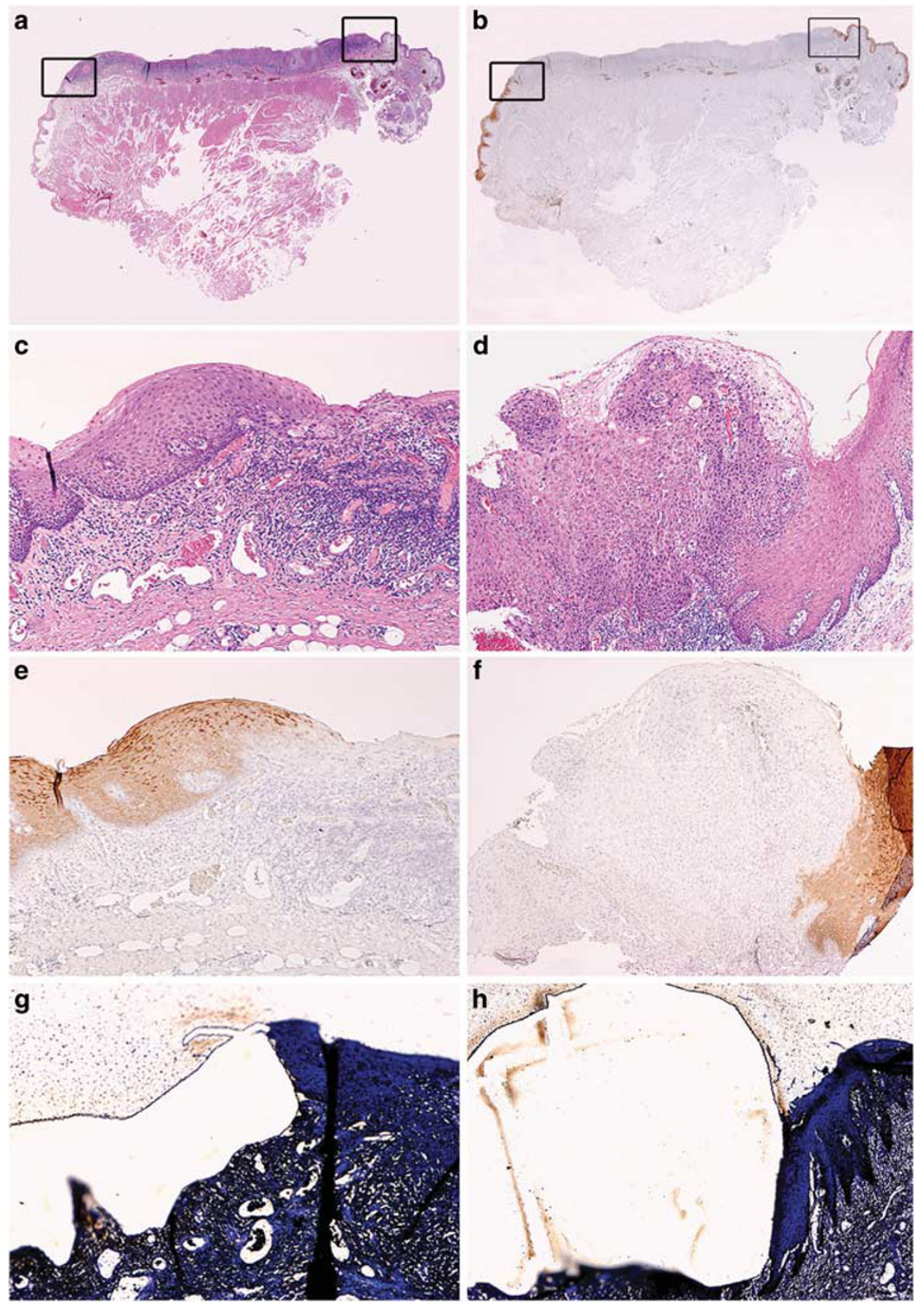

Figure 5 Laser microdissection from keratin $(\mathrm{K}) 13(+)$ or K13(-) epithelial lesion. (a, c, d) Hematoxylin and eosin (HE) stain, (b, e, f) immunoperoxidase stain for K13, hematoxylin counterstain. (g, h) areas taken by laser-capture microdissection (LMD), toluidine blue staining. Using LMD, we collected separately from the areas for $\mathrm{K} 13(+)(\mathbf{c}, \mathbf{e}$, g; left square in $\mathbf{a}$ and $\mathbf{b})$ and $\mathrm{K} 13(-)$ (d, f, h; right square in a and b) epithelial cells.

\section{Direct Sequencing for PCR Products}

To confirm that the RT-PCR products from the LMD samples were for K13 mRNA, we examined them by direct sequencing. All of the K13 PCR products from both $\mathrm{K} 13(+)$ and $\mathrm{K} 13(-)$ parts were verified to be the amplicon of K13 mRNA without any mutations. Partial sequencing data of $\mathrm{K} 13(-)$ samples are shown in Figure 6c.

\section{ISH for K13 mRNA}

As the definite expression of K13 mRNA was obtained even in the K13(-) CIS parts in $57 \%$ of the specimens examined, we confirmed the discrepancy by ISH for K13 mRNA in the serial sections. In $\mathrm{K} 13(+)$ normal epithelial parts, mRNA signals were detected in the cytoplasm of epithelial cells in the whole layers (Figure 7a-c). In contrast, very faint 


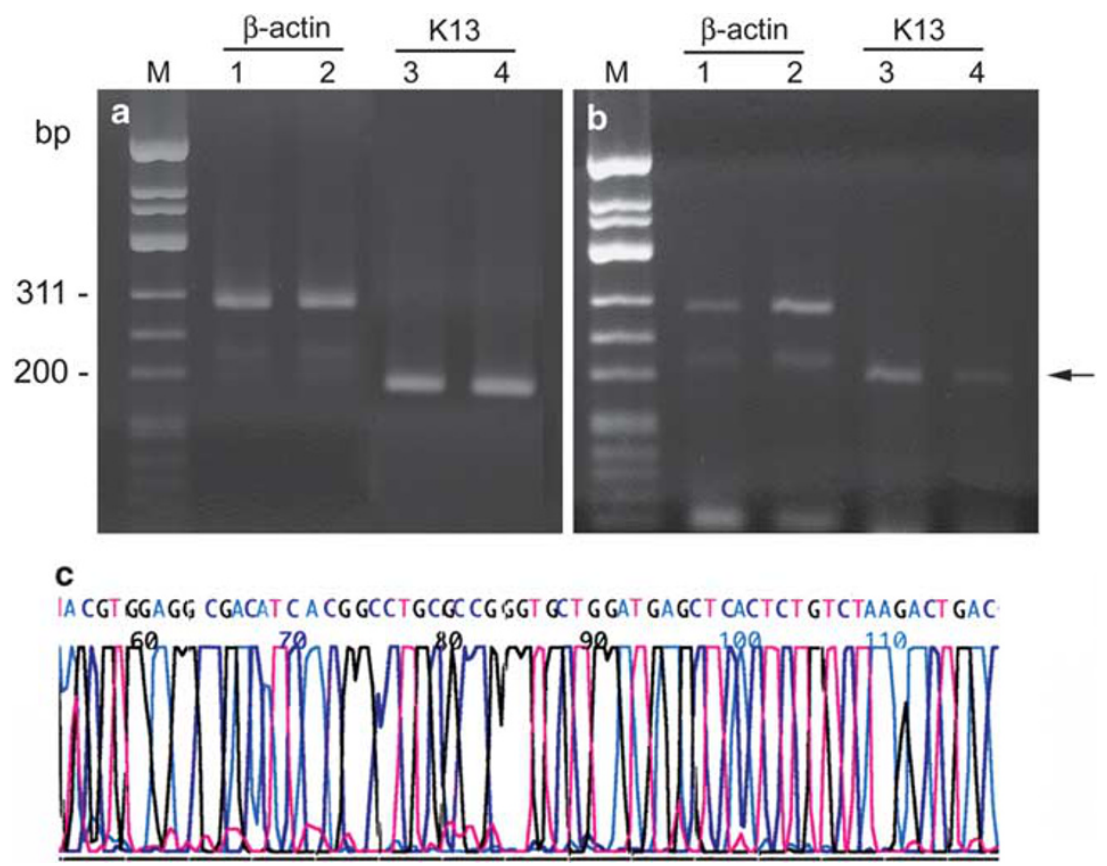

Figure 6 Reverse transcriptase (RT)-PCR for K13 from microdissected keratin (K)13(+) or K13(-) epithelial lesion in the same section and direct sequencing for PCR products. (a) Case 1, (b) case 2. (Lanes 1 and 2) 295-bp fragment of $\beta$-actin, (lanes 3 and 4) 195-bp fragment of K13. (Lanes 1 and 3) K13(+) normal epithelium, (lanes 2 and 4) K13(-) carcinoma in situ (CIS) lesion. In four cases, the strong band of K13 mRNA was detected in the K13(-) CIS region (a). On the other hand, in three cases, only a faint band was detected in the K13(-) epithelium, which was seen to correspond with immunohistochemical results (b). (c) Partial sequencing data of K13 PCR products corresponding to nucleotide number 687-735, Number NM153490.1, GeneBank. Both K13(+) and K13(-) PCR products gave the same sequencing results without any mutation.

mRNA signals for K13 were demonstrated in K13(-) CIS parts (Figure $7 \mathrm{~d}-\mathrm{f}$ ). The control sections using sense probes were consistently not positive for K13 mRNA (data not shown). Accordingly, ISH also supported the RT-PCR result that K13 mRNA was expressed in the 4/7 K13(-) CIS specimens.

\section{Discussion}

In the present study, we were successful in performing RT-PCR using RNA samples extracted from formalin-fixed, paraffin-embedded surgical materials of the 23 cases of oral CIS, which has enabled us to demonstrate the K13 transcription status in reference to their ISH and immunohistochemical data.

Our previous studies have paid attention to the immunohistochemical loss of K13 in the malignant transformation process of the oral mucosa from epithelial dysplasia to $\mathrm{CIS}^{2,4-7}$ In normal oral epithelium, K13 was mainly immunolocalized in the prickle cells, but not in the first or second basal cells. However, its expression mostly disappeared from the whole epithelial layer of CIS. ${ }^{4-7,9-11}$ The expression profiles for the keratin genes have previously been demonstrated by using ISH techniques in normal oral mucosa, ${ }^{19}$ and by showing their alteration statuses in oral epithelial lesions ${ }^{20}$ including oral epithelial dysplasia and SCC. ${ }^{8}$ In the present ISH study, we found that the mRNA signals for K13 remained to some extent, but did not completely disappear in the immunohistochemically K13(-) CIS foci, indicating that their translation was suppressed by some posttranscriptional modifications.

The gene transcription levels for K13 in the oral mucosal lesions have insufficiently been investigated by RT-PCR. ${ }^{21-24} \mathrm{Lv}$ et $a l^{24}$ demonstrated by RT-PCR of fresh tissue samples that mRNA levels for the K13 gene were 4.2-fold higher in oral normal tissues than in paired cancerous samples. However, the relationship between the gene transcriptional and protein expression levels is unclear, because epithelial cells or SCC cells were not selectively sampled in those studies. Thus, the present study is the first trial to compare the K13 gene transcription levels by RT-PCR using LMD-prepared pure epithelial samples with their histological levels by ISH in accurate references to immunohistochemical levels in the same areas. Although the enhanced immunolocalizations for CK16 in CIS cases corresponded to their definite mRNA levels, the loss of K13 immunopositivities seemed to be partly due to lowered K13 gene expression levels and partly due to unknown posttranslational events. Areas with gene expressions for such keratin subtypes as $\mathrm{K} 1, \mathrm{~K} 6, \mathrm{~K} 7, \mathrm{~K} 8$, and $\mathrm{K} 10$ have been reported to be wider than their protein ones in normal oral mucosa and salivary glands, ${ }^{25,26}$ salivary pleomorphic adenoma, ${ }^{25}$ oral 

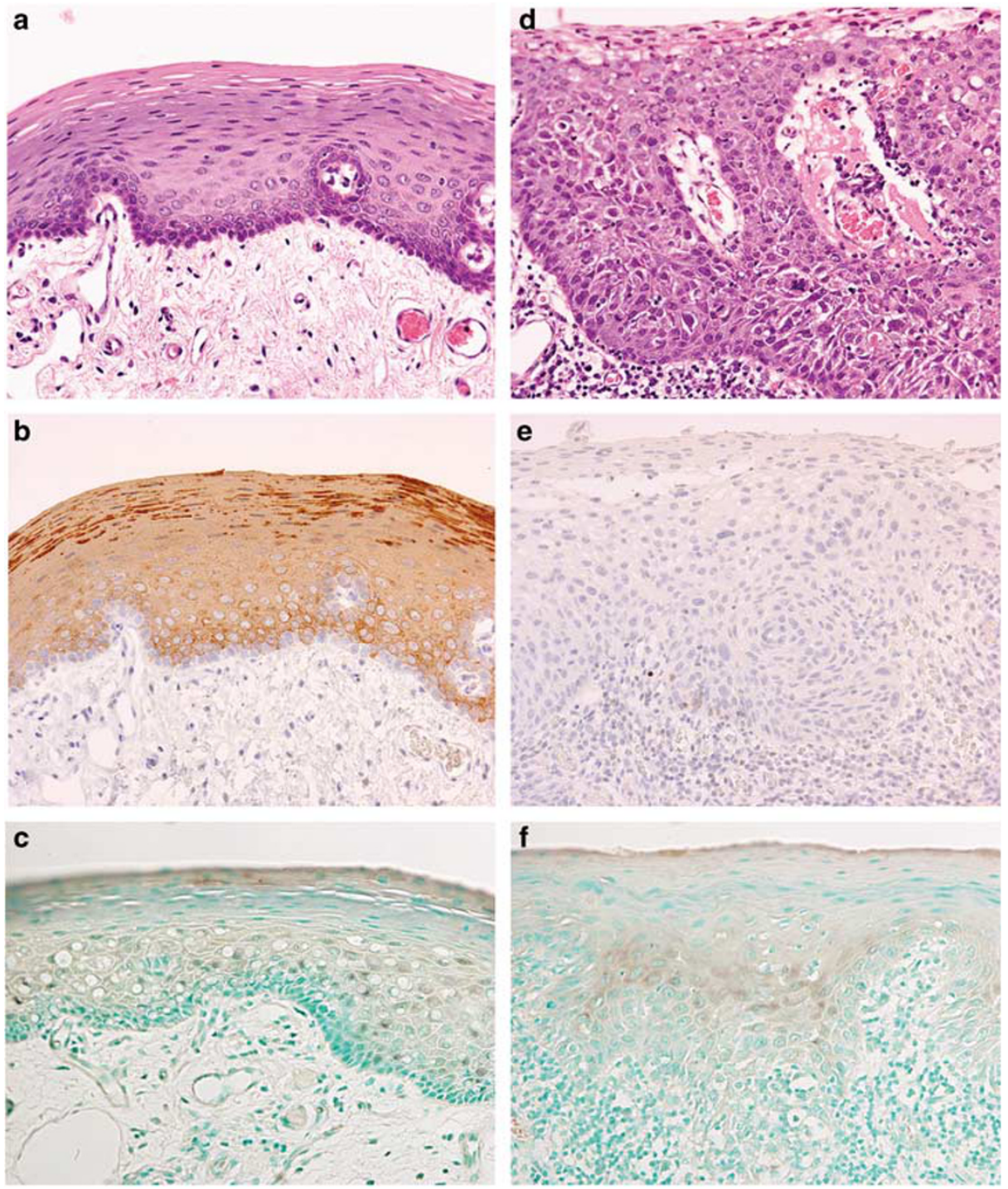

Figure 7 In-situ hybridization (ISH) for keratin (K)13 mRNA in K13(+) or K13(-) epithelial lesions. (a-c) K13(+) normal epithelium, (d-f) K13(-) carcinoma in situ (CIS) lesion, (a, d) Hematoxylin and eosin (HE) stain, (b, e) immunoperoxidase stain for K13, hematoxylin counterstain, (c, f) immuno-alkaline phosphatase stain for digoxigenin-labeled antisense K13 RNA probes, methylgreen counterstain. K13 mRNA is present in parabasal and prickle cells in normal epithelium (c). However, faint mRNA signals for K13 were also detected in the CIS cells, which did not show K13 immunopositivities (f). Original magnification: $\times 150$.

epithelial, ${ }^{26}$ or epidermal ${ }^{27}$ lesions. The tendencies were considered to be possible because the transcript forms might not always be translated to proteins due to some posttranscriptional regulations. ${ }^{21,25-27}$ In human skin epidermis, multiple microRNAs are thought to regulate gene expression of keratins during keratinocyte differentiation in vitro and in vivo. ${ }^{28}$ Therefore, it is speculated that microRNA-induced repression of the K13 gene translation might occur as posttranscriptional regulation in the oral keratinocytes.

The most important technical issue in the RNA extraction from paraffin sections in the present study was to prevent DNA contamination, which we had never experienced in RNA extraction from fresh samples. ${ }^{29-31}$ From our present experience, we would recommend the DNase I treatment as an essential step for RT-PCR for RNA samples extracted from paraffin sections to avoid DNA contamination. Another important technique to avoid false-positive mRNA bands was that we prepared primers to span an intron to generate different-sized products for RNA samples by excluding annealing to genomic DNA. ${ }^{23}$ In addition, the sizes of PCR products were designed to be less than $300 \mathrm{bp}$ for successful amplification, because RNA samples from formalin-fixed, paraffin-embedded tissues seemed to be degraded. Precipitative fixatives, such as ethanol and acetone, have been believed to produce more RT-PCR amplification products than cross-linking fixatives such as formaldehyde, ${ }^{29}$ though no differences have been documented between formalin- 
fixed tissue compared with fresh-frozen tissue in the qualitative expressions of such cancer-relevant genes, including that for FGF-R and HER-2. ${ }^{23}$ As we could obtain reasonable RNA samples from all of the 23 cases examined, it was concluded that the RNA extraction was possible in routine formalinfixed specimens. However, their qualities and yields of the RNA samples were different from case to case, which indicated RNase-free fixation and short storage time, in case RNase-free handlings are not expected. Therefore, it is important to control the time period from the surgical removal of tissue specimens to fixation in formalin, because RNA samples might undergo significant enzymatic degradation until the specimens are completely fixed and embedded. ${ }^{29,30,32}$

Needless to say, fresh samples are desirable for gene analysis, but fresh frozen tissues are not always available in clinical scenes. Formalin-fixed, paraffin-embedded tissue samples, which have been archived for decades through routine histopathological diagnostic activities in each hospital, are therefore regarded as a valuable resource, along with clinical information, for further genetic or molecular investigations towards diagnostic, retrospective, and investigative purposes if DNA or RNA samples are able to be recovered from them. The great advantage of paraffin sections is their preserved tissue architecture, which is a prerequisite for histopathological diagnostic confirmation and which allows a wide variety of approaches for further clinicopathological studies. In addition, application of laser microdissection may help in sampling carefully targeted cells for accurate RT-PCR results. Thus, these combined techniques have converted formalinfixed, paraffin-embedded materials from mere routine diagnostic subjects to important treasure minelike research resources for future pathological research.

\section{Acknowledgement}

This work was supported in part by Grants-in-Aid for Scientific Research from the Japan Society for the Promotion of Science and from the Ministry of Education, Science, Sports and Culture, Japan.

\section{Disclosure/conflict of interest}

The authors declare no conflict of interest.

\section{References}

1 Gala N, Pilch BZ, Sidransky D, et al. Epithelial precursor lesions In: Barnes L, Eveson JW, Reichart, P Sindransky D, (eds). Pathology and Genetics of Head and Neck Tumours. World Health Organization Classification of Tumours, IARC Press: Lyon, 2005, pp 177-179.
2 Lindberg K, Rheinwald JG. Suprabasal 40 kd keratin (K19) expression as an immunohistologic marker of premalignancy in oral epithelium. Am J Pathol 1989;134:89-98.

3 Sawaf MH, Ouhayoun JP, Shabana AH, et al. Cytokeratin expression in human tongue epithelium. Am J Anat 1990;189:155-166.

4 Shinohara M, Hiraki A, Ikebe T, et al. Immunohistochemical study of desmosomes in oral squamous cell carcinoma correlation with cytokeratin and E-cadherin staining, and with tumour behaviour. J Pathol 1998;184:369-381.

5 Kobayashi T, Maruyama S, Cheng J, et al. Histopathological varieties of oral carcinoma in-situ: Diagnosis aided by immunohistochemistry dealing with the second basal cell layer as the proliferating center of oral mucosal epithelia. Pathol Int 2010;60:156-166.

6 Mikami T, Cheng J, Maruyama S, et al. Emergence of keratin 17 vs. loss of keratin 13: their reciprocal immunohistochemical profiles in oral carcinoma in situ. Oral Oncol 2011;47:497-503.

7 Sawaf MH, Ouhayoun JP, Forest N. Cytokeratin profiles in oral epithelia: a review and a new classification. J Biol Buccale 1991;19:187-198.

8 Kuruc N, Leube RE, Moll I, et al. Synthesis of cytokeratin 13, a component characteristic of internal stratified epithelia, is not induced in human epidermal tumors. Differentiation 1989;42:111-123.

9 Ogden GR, Chisholm DM, Adi M, et al. Cytokeratin expression in oral cancer and its relationship to tumor differentiation. J Oral Pathol Med 1993;22:82-86.

10 Bloor BK, Seddon SV, Morgan PR. Gene expression of differentiation-specific keratins in oral epithelial dysplasia and squamous cell carcinoma. Oral Oncol 2001;37:251-261.

11 Guang HE, Wei-Neng FU, Guang-Bin QIU, et al. Studies of the deletion and expression of cytokeratin 13 gene in laryngeal squamous cell carcinoma. Acta Genetica Sinica 2002;29:390-395.

12 Yanagawa $\mathrm{T}$, Yoshida $\mathrm{H}$, Yamagata $\mathrm{K}$, et al. Loss of cytokeratin 13 expression in squamous cell carcinoma of the tongue is a possible sign for local recurrence. J Exp Clin Cancer Res 2007;26:215-220.

13 Ohta K, Ogawa I, Ono S, et al. Histopathological evaluation including cytokeratin 13 and Ki-67 in the border between Lugol-stained and -unstained areas. Oncol Rep 2010;24:9-14.

14 Sakamoto K, Aragaki T, Morita KI, et al. Downregulation of keratin 4 and keratin 13 expression in oral squamous cell carcinoma and epithelial dysplasia: a clue for histopathogenesis. Histopathology 2011;58: 531-542.

15 Sesterhenn AM, Mandic R, Dunne AA, et al. Cytokeratins 6 and 16 are frequently expressed in head and neck squamous cell carcinoma cell lines and fresh biopsies. Anticancer Res 2005;25:2675-2680.

16 Leigh IM, Navsaria H, Purkis PE, et al. Keratins (K16 and K17) as markers of keratinocyte hyperproliferation in psoriasis in vivo and in vitro. $\mathrm{Br} \mathrm{J}$ Dermatol 1995;133:501-511.

17 Komine M, Rao LS, Kaneko T, et al. Inflammatory versus proliferative processes in epidermis. Tumor necrosis factor alpha induces K6b keratin synthesis through a transcriptional complex containing NFkappa B and C/EBPbeta. J Biol Chem 2000;275:32077-32088.

18 Ida-Yonemochi $\mathrm{H}$, Ahsan MS, Saku T. Differential expression profiles between $\alpha$-dystroglycan and 
integrin $\beta 1$ in ameloblastoma: two possible perlecan signalling pathways for cellular growth and differentiation. Histopathology 2011;58:234-245.

19 Bloor BK, Seddon SV, Morgan PR. Gene expression of differentiation-specific keratins (K4, K13, K1 and K10) in oral non-dysplastic keratoses and lichen planus. J Oral Pathol Med 2000;29:376-384.

20 Ohkura S, Kondoh N, Hada A, et al. Differential expression of the keratin-4, -13, -14, -17 and transglutaminase 3 genes during the development of oral squamous cell carcinoma from leukoplakia. Oral Oncol 2005;41:607-613.

$21 \mathrm{Su} \mathrm{L}$, Morgan PR. Discrepancies between keratin mRNA and protein expression in mucosal and glandular epithelia by in situ hybridization and immunohistochemistry. Cell Vision J Analyt Morphol 1994; 1:146-153.

22 Leethanakul C, Patel V, Gillespie J, et al. Gene expression profiles in squamous cell carcinomas of the oral cavity: use of laser capture microdissection for the construction and analysis of stage-specific cDNA libraries. Oral Oncol 2000;36:474-483.

23 Specht K, Richter T, Müller U, et al. Quantitative gene expression analysis in microdissected archival formalin-fixed and paraffin-embedded tumor tissue. Am J Pathol 2001;158:419-429.

24 Lv XZ, Chen WT, Zhang CP. Expression and significance of cytokeratin 13 gene in oral squamous cell carcinoma]. Shanghai Kou Qiang Yi Xue 2005;14: 378-381.
$25 \mathrm{Su} \mathrm{L}$, Morgan PR, Harrison D, et al. Expression of keratin mRNAs and proteins in normal salivary epithelia and pleomorphic adenomas. J Pathol 1993;171:173-181.

$26 \mathrm{Su} \mathrm{L}$, Morgan PR, Lane EB. Protein and mRNA expression of simple epithelial keratins in normal, dysplastic and malignant oral epithelia. Am J Pathol 1994;145:1-10.

27 Tyner AL, Fuchs E. Evidence for post-transcriptional regulation of the keratins expressed during hyperproliferation and malignant transformation in human epidermis. J Cell Biol 1986;103:1945-1955.

28 Hildebrand J, Rütze $\mathrm{M}$, Walz $\mathrm{N}$, et al. A comprehensive analysis of microRNA expression during human keratinocyte differentiation in vitro and in vivo. J Invest Dermatol 2011;131:20-29.

29 Rupp GM, Locker J. Purification and analysis of RNA from paraffin-embedded tissues. Biotechniques 1988; 6:56-60.

30 Stanta G, Schneider C. RNA extracted from paraffinembedded human tissues is amenable to analysis by PCR amplification. Biotechniques 1991;11:304-308.

31 Goldsworthy SM, Stockton PS, Trempus CS, et al. Effects of fixation on RNA extraction and amplification from laser capture microdissected tissue. Mol Carcinog 1999;25:86-91.

32 Finke J, Fritzen R, Ternes P, et al. An improved strategy and a useful housekeeping gene for RNA analysis from formalin-fixed, paraffin-embedded tissues by PCR. Biotechniques 1993;143:448-453. 